\title{
Treatment of Digital Ulcers and Reflux Oesophagitis in a Patient with Systemic Sclerosis: Increased Risk of Hepatotoxicity due to a Potential Drug-drug Interaction Between Bosentan and Vonoprazan
}

Ryoko KIMURA ${ }^{1}$, Kazunari SUGITA ${ }^{2}$, Takaaki SUGIHARA ${ }^{3}$, Hajime ISOMOTO ${ }^{3}$ and Osamu YAMAMOTO $^{1}$

${ }^{1}$ Division of Dermatology, Department of Medicine of Sensory and Motor Organs, Faculty of Medicine, Tottori University, 36-1 Nishi-cho, Yonago 683-8503, ${ }^{2}$ Division of Dermatology, Department of Internal Medicine, Faculty of Medicine, Saga University, Saga, and ${ }^{3}$ Division of Medicine and Clinical Science, Department of Gastroenterology and Nephrology, Tottori University Faculty of Medicine, Yonago, Tottori, Japan. E-mail: m0641023@tottori-u.ac.jp

Accepted Oct 25, 2021; Epub ahead of print Oct 25, 2021

Bosentan is an endothelin receptor antagonist that has been approved for treatment of systemic sclerosis (SSc)associated pulmonary hypertension and digital ulcers related to SSc. One of the major adverse drug reactions of bosentan is hepatotoxicity (1). In this report, we describe a patient with SSc who developed severe liver dysfunction during concomitant treatment with bosentan and vonoprazan fumarate. After a temporary cessation of treatment, liver function tests normalized, and drug treatment with bosentan in combination with lansoprazole could be resumed without further signs of liver injury.

\section{CASE REPORT}

A 56-year-old Japanese man who had been taking $30 \mathrm{mg}$ lansoprazole once daily for reflux oesophagitis was referred to our clinic for evaluation of a 5-year history of digital swelling and Raynaud's phenomenon, followed by ischaemic ulcers developing in several fingertips. Physical examination revealed diffuse swelling of the hands and fingers, as well as nail fold bleeding in dermoscopic observation (Fig. 1a, b). In addition, the left third fingertip and the right fifth fingertip showed painful digital ulcers, and the left second fingertip showed a digital pitting scar (Fig. 1c, d). Histopathological examination of the dorsal side of the right third finger revealed thick collagen bundles and a lack of sweat glands in the dermis (Fig. 1e, f). Immunological test results were as follows: positive antinuclear antibody (ANA) titre at 1:1280 (centromere type) (normal range $<1: 40$ ), positive anticentromere antibody at $171 \mathrm{IU} / \mathrm{ml}$ (normal range $<10$ ), and negative for anti-topoisomerase antibody. The patient was diagnosed with limited cutaneous SSc. Treatment with bosentan was initiated at a starting dose of $62.5 \mathrm{mg}$ twice daily for 4 weeks, and the final maintenance dose was $125 \mathrm{mg}$ twice daily. Seven months after initiation of the treatment, no new digital ulcers were observed and the pre-existing painful ulcers were improved (Fig. 1g, h). While the skin lesions remained stable with treatment, the patient's reflux oesophagitis worsened, and lansoprazole was switched to vonoprazan fumarate, a potassium-competitive acid blocker (P$\mathrm{CAB})$. After 6 months, the patient had several abnormal results in liver function tests: aspartate aminotransferase (AST) (490 U/1; normal, 13-33), alanine aminotransferase (ALT) (524 U/1; normal, 8-42), alkaline phosphatase (ALP) (315 U/1; normal, 115-359), $\gamma$-glutamyl transpeptidase ( $\gamma$-GTP) (335 U/1; normal, 13-64), cholinesterase (357 U/1; normal, 240-486), lactate dehydrogenase (LDH) (714 U/1; normal, 119-229) and total bilirubin (0.6 mg/dl; normal, 0.4-1.5). Hepatitis $\mathrm{B}$ was positive for $\mathrm{HBsAb}$, but negative for $\mathrm{HBsAg}, \mathrm{HBcAb}$ and $\mathrm{HBV} \mathrm{DNA}$. Virological tests for hepatitis $\mathrm{A}$ and $\mathrm{C}$ were negative. Cytomegalovirus antigenaemia testing was negative. Epstein-Barr virus viral-capsid antigen (VCA)-IgM was negative, and VCA-IgG was positive. Roussel Uclaf Causality Assessment Method (RUCAM) score was 8, which fell in the group of "probable" for both bosentan and vonoprazan fumarate (2). One month after discontinuation of treatment with bosentan and vonoprazan fumarate, his liver dysfunction was restored to normal levels and hardening of the skin of the fingers worsened. We decided to cautiously resume treatment with bosentan after getting informed consent from the patient. Since the patient only had mild symptoms of reflux oesophagitis, we re-administered $30 \mathrm{mg}$ lansoprazole daily and bosentan at a dose of $62.5 \mathrm{mg}$ once daily for 1 week, then $62.5 \mathrm{mg}$ twice daily for 2 weeks, and the final maintenance dose of bosentan of was $125 \mathrm{mg}$ twice daily. Liver function normalized completely within 1 month of resuming bosentan and lansoprazole combination therapy, and there were no signs of liver abnormalities or new onset digital ulcers after a further 3 months.
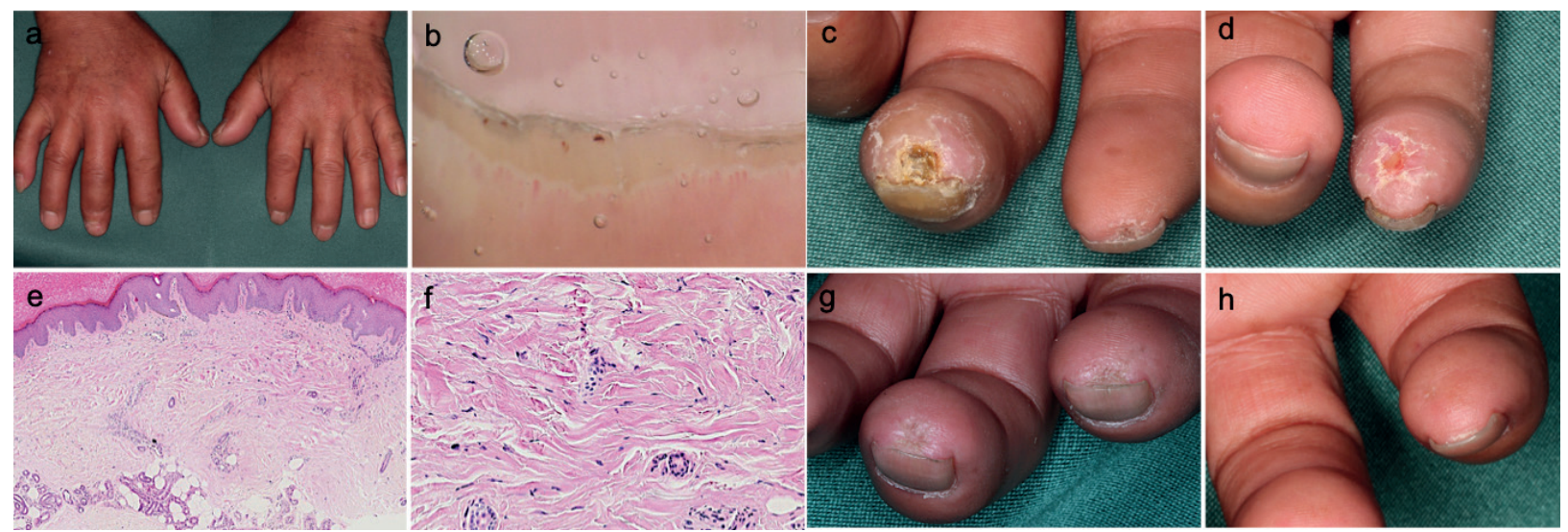

Fig. 1. (a) Digital oedema at the first visit. (b) Dermoscopic findings of nail fold bleeding. Digital ulcers on (c) the third fingertip of the left hand and (d) the fifth fingertip of the right hand at the first visit. (e) A biopsy specimen from the dorsal side of the right third finger (haematoxylin and eosin (H\&E), original magnification $\times 100)$. ( $f)$ Thickened collagen bundles in the dermis $(H \& E, \times 200)$. $(g)$ The third fingertip of the left hand and $(h)$ fifth fingertip of the right hand after introduction of bosentan. 


\section{DISCUSSION}

Management of digital ulcers and oesophageal disease is essential in patients with SSc. Bosentan, an endothelin receptor-1 antagonist, is approved for treatment of pulmonary arterial hypertension and Raynaud's phenomenon as well as skin fibrosis in patients with SSc. Bosentan can reduce the risk of new digital ulcers in these patients. Recent studies have shown that bosentan is also effective for treating established digital ulcers (3). Vonoprazan fumarate is a first-in-class potassium-competitive acid blocker that has been approved in some Asian countries, such as Japan, China and Singapore, but the medication is unavailable in Europe (4-6). In the current case, the digital ulcers were reduced in size and no new onset digital ulcers were observed after starting bosentan treatment. Interestingly, abnormal liver function tests were first observed after changing from lansoprazole to vonoprazan fumarate and normalized after a treatment pause and resumption of therapy with bosentan in combination with lansoprazole.

Bosentan is mainly metabolized by cytochrome P450 (CYP) 2C9 and CYP3A4 and, to a lesser extent, CYP2C19. It has been reported that inhibition of these enzymes in the course of drug-drug-interactions will likely lead to large increases in plasma concentrations of bosentan (7). Lansoprazole is mainly metabolized by CYP2C19 and only partly by CYP3A4, while vonoprazan fumarate is mainly metabolized by CYP3A4 and partly by CYP2B6, CYP2C19 and CYP2D6 (4). Furthermore, it has been demonstrated that vonoprazan fumarate may inhibit both CYP3A4 (8) and CYP2C19, which theoretically could result in elevated plasma concentrations of bosentan, increasing the risk of drug-induced liver injury. Patients with SSc frequently develop reflux oesophagitis, and proton pump inhibitors (PPIs) are often prescribed. The majority of PPIs are metabolized by the liver, mainly through CYP2C19 and CYP3A4 pathways (9). Furthermore, most PPIs show the potential of inhibiting CYP2C19, with omeprazole and esomeprazole being the most potent CYP2C19 inhibitors while rabeprazole and pantoprazole are considered being the weakest inhibitors among traditional PPIs (10). Studies in pooled human liver microsomes show that lansoprazole is not a metabolismdependent inhibitor of CYP2C19 (11) and only a very weak inhibitor of CYP3A4 (12). In contrast, vonoprazan fumarate appears to inhibit CYP2C19 in the same order of magnitude as esomeprazole (13). Therefore, not only lansoprazole, but also rabeprazole and pantoprazole, can be potential drug candidates in combination with bosentan.

In this case, RUCAM score of vonoprazan fumarate was "probable" rather than "highly probable". Although vonoprazan fumarate has been developed as a $\mathrm{P}-\mathrm{CAB}$ with low hepatotoxicity (14), hepatotoxicity has been reported in $0.6-2 \%$ of patients after administration of vonoprazan fumarate in a randomized controlled trial (15). Thus, the possible relationship between vonoprazan fumarate and hepatotoxicity would be considered in the current case.
Measurement of plasma concentrations of bosentan before and after vonoprazan fumarate administration might have strengthened the hypothesis that the hepatotoxicity was caused by an interaction between bosentan and vonoprazan fumarate. Since Asians inherit a large proportion of the CYP2C19 poor metabolizers gene in relation to Caucasians (16), CYP2C19 polymorphism may influence the present hepatotoxicity.

In conclusion, the current case report shows that caution should be exercised when bosentan is concomitantly used with vonoprazan fumarate, due to the risk of drug-drug interactions caused by the inhibitory effect of vonoprazan fumarate on CYP3A4. Lansoprazole is primarily metabolized by CYP2C19 and only partly by CYP3A4 and is not a metabolism-dependent inhibitor of CYP2C19 and only a very weak inhibitor of CYP3A4; therefore, lansoprazole may be used concomitantly with bosentan. Further investigations are needed to determine whether the combined therapy of bosentan with various other PPIs is safe in patients with systemic scleroderma who have reflux oesophagitis.

The authors have no conflicts of interest to declare.

\section{REFERENCES}

1. Eriksson C, Gustavsson A, Kronvall T, Tysk C. Hepatotoxicity by bosentan in a patient with portopulmonary hypertension: a case-report and review of the literature. J Gastrointestin Liver Dis 2011; 20: 77-80.

2. Danan G, Benichou C. Causality assessment of adverse reactions to drugs - I. A method based on the conclusions of international consensus meetings: application to druginduced liver injuries. J Clin Epidemiol 1993; 46: 1323-1330.

3. Tingey T, Shu J, Smuczek J, Pope J. Meta-analysis of healing and prevention of digital ulcers in systemic sclerosis. Arthritis Care Res 2013; 65: 1460-1471.

4. Echizen $H$. The First-in-class potassium-competitive acid blocker, vonoprazan fumarate: pharmacokinetic and pharmacodynamic considerations. Clin Pharmacokinet 2016; 55: 409-418.

5. Herszényi L, Bakucz T, Barabás L, Tulassay Z. Pharmacological approach to gastric acid suppression: past, present, and future. Dig Dis 2020; 38: 104-111.

6. Scarpignato $\mathrm{C}$, Hunt $\mathrm{RH}$. The potential role of potassium-competitive acid blockers in the treatment of gastroesophageal reflux disease. Curr Opin Gastroenterol 2019; 35: 344-355.

7. Srinivas NR. Clinical drug-drug interactions of bosentan, a potent endothelial receptor antagonist, with various drugs: physiological role of enzymes and transporters. Gen Physiol Biophys 2016; 35: 243-258.

8. Wang Y, Wang C, Wang S, Zhou Q, Dai D, Shi J, et al. Cytochrome P450-based drug-drug interactions of vonoprazan in vitro and in vivo. Front Pharmacol 2020; 11: 53.

9. Furuta T, Shirai N, Sugimoto M, Nakamura A, Hishida A, Ishizaki T. Influence of CYP2C19 pharmacogenetic polymorphism on proton pump inhibitor-based therapies. Drug Metab Pharmacokinet 2005; 20: 153-167.

10. Zvyaga T, Chang SY, Chen C, Yang Z, Vuppugalla R, Hurley J, et al. Evaluation of six proton pump inhibitors as inhibitors of various human cytochromes P450: focus on cytochrome P450 2C19. Drug Metab Dispos 2012; 40: 1698-1711.

11. Ogilvie BW, Yerino P, Kazmi F, Buckley DB, Rostami-Hodjegan $A$, Paris BL, et al. The proton pump inhibitor, omeprazole, but not lansoprazole or pantoprazole, is a metabolism-dependent inhibitor of CYP2C19: implications for coadministration with clopidogrel. Drug Metab Dispos 2011; 39: 2020-2033. 
12. Li X-Q, Andersson TB, Ahlström M, Weidolf L. Comparison of inhibitory effects of the proton pump-inhibiting drugs omeprazole, esomeprazole, lansoprazole, pantoprazole, and rabeprazole on human cytochrome P450 activities. Drug Metab Dispos 2004; 32: 821-827.

13. Funakoshi R, Tomoda $Y$, Kudo T, Furihata K, Kusuhara H, Ito K. Effects of proton pump inhibitors, esomeprazole and vonoprazan, on the disposition of proguanil, a CYP2C19 substrate, in healthy volunteers. Br J Clin Pharmacol 2019; 85: 1454-1463.

14. Oshima T, Miwa H. Potent potassium-competitive acid blockers: a new era for the treatment of acid-related diseases. J
Neurogastroenterol Motil 2018; 24: 334-344.

15. Ashida K, Sakurai $Y$, Hori T, Kudou K, Nishimura A, Hiramatsu N, et al. Randomised clinical trial: vonoprazan, a novel potassium-competitive acid blocker, vs. lansoprazole for the healing of erosive oesophagitis. Aliment Pharmacol Ther 2016; 43: 240-251.

16. Shimizu T, Ochiai H, Asell F, Shimizu H, Saitoh R, Hama $Y$, et al. Bioinformatics research on inter-racial difference in drug metabolism I. Analysis on frequencies of mutant alleles and poor metabolizers on CYP2D6 and CYP2C19. Drug Metab Pharmacokinet 2003; 18: 48-70. 